\title{
Ihre Beiträge in der FTR 2012
}

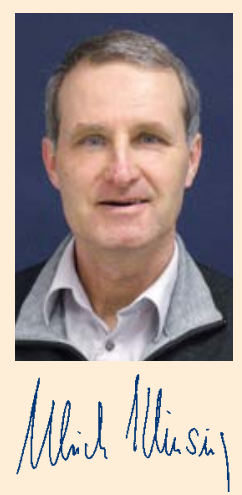

Liebe Kolleginnen und Kollegen,

die erste Ausgabe des Jahres bietet eine gute Gelegenheit für einen kurzen Rückblick auf die Entwicklung unserer Zeitschrift im vergangenen Jahr, was natürlich gleichzeitig Perspektiven für das Jahr 2012 eröffnet.

Die Zeitschrift Flugmedizin Tropenmedizin Reisemedizin ist bei einer Auflage von über 5000 Exemplaren weiter gewachsen. Interessant ist dabei auch die starke Zunahme der Zugriffszahlen im Internetangebot. Im ersten Halbjahr konnten bereits über 14000 Aufrufe gezählt werden. Das waren zu diesem Zeitpunkt bereits über doppelt so viel Zugriffe wie im Gesamtjahr 2010! Dies liegt sicher zum einen an den attraktiven wissenschaftlichen Beiträgen mit spannenden Themen aus unterschiedlichen Fachgebieten unter dem Blickwinkel der Reisemedizin. Zum anderen aber auch an der gesunden Mischung von wissenschaftlichen Übersichts- und Originalartikeln einerseits und interessanten Beiträgen in den weiteren Rubriken Magazin, Journal-Club, Kasuistik, Forum der Industrie und Feuilleton andererseits, die ja ebenfalls ganz wesentliche Elemente unserer Zeitschrift darstellen. Auch die Gesellschaftsseiten mit ihren Informationsmöglichkeiten über die eigene, aber auch über alle anderen in dieser Zeitschrift vertretenen Gesellschaften tragen zur Attraktivität für den Leser bei.

Bewährt hat sich auch die Unterstützung der Redaktion durch das Editorial Board, dem insbesondere alle Original- und Übersichtsarbeiten in einem Peer-Review-Verfahren zur inhaltlichen Überprüfung vorgelegt werden. Diese Begutachtung hat das Ziel, die Qualität der wissenschaftlichen Beiträge zu erhöhen und damit dazu beizutragen, dass die Artikel dieser Rubrik die Anforderungen an eine wissenschaftliche Veröffentlichung erfüllen. Wenn notwendig, werden Verbesserungsvorschläge unterbreitet.

In seiner letzten Sitzung Ende vergangenen Jahres hat das Editorial Board Überlegungen dazu angestellt, wie man Sie als Leser dieser Zeitschrift noch mehr in die inhaltliche Gestaltung der Zeitschrift einbinden kann. Hier sollen sich nicht nur die bereits wissenschaftlich tätigen Kollegen angesprochen fühlen, die wir selbstverständlich zur Einreichung von Manuskripten mit interessanten und aktuellen Themen für Übersichts- und Originalartikel in der FTR auffordern. Vielmehr möchten wir auch weniger wissenschaftlich routinierte Leser dazu motivieren, sich aktiv an der Gestaltung der Zeitschrift zu beteiligen. Die Rubriken Magazin und Feuilleton bieten Platz für informative, spannende oder auch unterhaltsame Themen außerhalb der strengen wissenschaftlichen Autorenvorgaben. In diesen Artikeln sollte allerdings ein klarer medizinischer Bezug erkennbar sein. Ein Reisebericht alleine reicht für eine Veröffentlichung nicht aus.

Ein gutes Beispiel für derartige Artikel ist der Beitrag im Reise-Feuilleton „Ferne Inseln im Südpazifik, Segelreise mit medizinischen Akzenten“. Dieser Artikel beschreibt neben einer beeindruckenden Reise eine Reihe von medizinischen Problemen, die sich bei einer solchen Unternehmung einstellen können. Die Leserbeiträge müssen natürlich nicht immer so spektakulär und umfangreich sein. Also, liebe Leser, beteiligen Sie sich an der Gestaltung unserer Zeitschrift. Jetzt wünsche ich Ihnen zunächst einmal bei der Lektüre der ersten Flugmedizin Tropenmedizin Reisemedizin im Jahr 2012 viel Vergnügen. 